Session 2363

\title{
A Graphical Post-Processor for an Educational CNC Code Generator
}

\author{
Slade Gellin, Anthony Hotchkiss \\ Department of Technology \\ Buffalo State College \\ Buffalo NY 14222
}

\begin{abstract}
The authors have developed a post-processor with a graphical user interface for use with a CNC code generator that was originally intended to operate one particular vertical milling machining center. The CNC code-generator, VAL-CAM, was developed by one of the authors for teaching part of a CAD/CAM course for engineering technology students. In order to make the CNC code more universally applicable, a post-processor has been written as a stand-alone program. The program not only converts the current $\mathrm{CNC}$ code to operate other machine tools, but also allows the addition of machine tool functions that are not included in the existing $\mathrm{CNC}$ code. This will allow engineering technology students to use a variety of CNC milling machines that have recently been, and may at some future time be, installed at Buffalo State College.

The program was written in Visual Basic to run under the Microsoft Windows environment, and hence on any PC platform. Modules have been written for existing CNC milling machines. The modules allow for capabilities of new machines to be added above and beyond those capabilities already incorporated. For instance, the machine for which the VAL-CAM program was originally written does not have an automatic tool changer, whereas one of the recently installed machine tools does have such a tool changer. The program module for this new machine allows the user to specify the codes that are used by the new machine that will effect an automatic change of tool. A variety of standard and non-standard codes are also provided so that the user can tailor the code to operate most new machine tools.
\end{abstract}

The significance of this project is that the generality and modularity of the post-processor lends itself to utilization in similar laboratories at other educational facilities.

\section{Introduction and Problem Statement}

A CNC code generator, VAL-CAM ${ }^{1,2,3}$, was developed by one of the authors for teaching part of a CAD/CAM course for engineering technology students. This program is couched as an attachment to AutoCAD and is written in AutoLisp, a command language affiliated with AutoCAD. The program used geometric data from a drawing currently on the AutoCAD screen, and, after the user supplied choices for various tooling and milling options, produced CNC code for one particular vertical milling machining center, as well as a graphical representation on the AutoCAD screen of the milling process. This process provided an instructional tool for learning 
about $\mathrm{CNC}$ machining in general and reading and understanding $\mathrm{CNC}$ coding in particular. How well students learn these concepts is assessed through exams and projects.

Recently, the Department of Technology at Buffalo State College has purchased and installed new milling machines each with different capabilities and/or options. The CNC codes required for these machines reflect their individual capabilities. In order to effectively use these machines in the course, thus giving students a greater variety of $\mathrm{CNC}$ machining experience, it was necessary to obtain software to facilitate the process from drawing to machine part.

A wide variety of CAD/CAM software is available commercially. The scope and complexity of these programs vary widely. Those that have broad applicability would require many hours of training that would take significant time away from the rest of the course curriculum. All had price tags well beyond what the Department of Technology could afford.

It was at this point that it was decided to write the necessary code in-house. In the course of determining the best way of doing this task, the authors realized an opportunity for manufacturing engineering technology and engineering education research. Accordingly, the project is now considered a solution to the following problem statement:

How does one best develop, utilize and assess the impact of a piece of software that satisfies both a technical and pedagogical need in an established CAD/CAM course?

The authors believed that the necessary enhancements to VAL-CAM should be incorporated in a multiple path approach. The two primary paths were in (1) CNC coding for the various machines, and (2) functional and graphical enhancements of the program. After much discussion, it was decided that the two paths should be housed in separate programs. In order to accomplish this, VAL-CAM would now have as its output a set of "generic" instructions for process and tool selection, as well as the appropriate geometric data, while a new, stand-alone post-processor, tentatively named BSC-POST, would take this output and, after the user has selected a machine and its particular features to be used for the intended process, would generate the appropriate $\mathrm{CNC}$ code for that machine.

This paper describes the development, features, implementation and significance of BSC-POST, a graphical post-processor for an educational CNC code generator.

\section{Objectives}

The objectives of this research are as follows:

- To develop a post-processor to work in tandem with VAL-CAM that will automate code generation for CNC milling machines currently in use in laboratories in the Department of Technology at Buffalo State College.

- That this post-processor be easy to learn and use within the confines of a required, existing $\mathrm{CAD} / \mathrm{CAM}$ course.

- That efficient utilization of the post-processor enhances the overall learning experience in the CAD/CAM course. 
- That the design of the program be such that it is expandable should Buffalo State College purchase additional $\mathrm{CNC}$ milling machines.

- That program improvement implementation provides opportunities for senior and master's level projects.

- That this project be accomplished at little or no cost to Buffalo State College.

To measure the extent to which these objectives are being met, the authors will use the following assessment tools and/or criteria:

- If VAL-CAM and BSC-POST together can successfully produce a part based on an AutoCAD drawing, then the first objective above will be satisfied.

- The effort required to incorporate data associated with subsequent machines will be measured in man-hours to determine if the program is readily expandable.

- An undergraduate student assisted with this project as part of an Independent Study course. A graduate student is currently working on improvements to VAL-CAM as part of a master's project.

- To assess whether the post-processor is easy to learn and use, and if it is enhancing the learning experience in the $\mathrm{CAD} / \mathrm{CAM}$ course, a questionnaire about the programs will be given to the students in the course. They will be asked to respond to positive statements about the programs on a Likert scale ranging from Strongly Agree to Strongly Disagree. This questionnaire is in development as of this writing, but will be available by semester end.

- Projects and exams will continue to measure the students' understanding of CNC coding.

- The project costs will be measured.

\section{Discussion}

The primary goal of the development of BSC-POST is stated in the problem statement above. To this end, the authors had many discussions on the capabilities and formatting that the program should have. An overview of the program features and utilization is given in the Appendix. Great importance was given to keeping the program as simple, yet thorough, as possible, in order to maximize its utilitarian value and minimize the training time.

Early tests of the program indicated that BSC-POST accomplished its technical objective of writing CNC code for each of the milling machines currently available to the Department of Technology. Furthermore, once the code was debugged for the first machine, subsequent machine incorporation went faster, at about $25 \%$ of the time needed for the first machine.

An "alpha" version of the program was shown to students that had previously taken the $\mathrm{CAD} / \mathrm{CAM}$ course in order to determine if the design of the program was conducive to meeting the educational objectives described above. These students generally had a favorable reaction to the program on issues of user-friendliness and their ability to learn the program quickly. Many of them (about 80\%) expressed the opinion that if the program were available when they had taken the $\mathrm{CAD} / \mathrm{CAM}$ course that they would have had an enhanced learning experience in the use of CNC programming. While these students had more of a "hindsight" perspective than the target group of students that would potentially be exposed to $\mathrm{CNC}$ programming for the first time, it was believed that their comments and suggestions carried enough weight to indicate that 
the general approach taken was correct, though modifications to individual features in the program would be necessary to maximize meeting the educational objectives stated above.

As noted above, a questionnaire is in development to elicit student perceptions on the effectiveness of BSC-POST in facilitating their understanding of the applications of CNC programming. Some typical statements to be evaluated by students would be:

"BSC-POST was easy to learn."

"BSC-POST enabled me to understand the CNC code output when placed along-side the input from VAL-CAM."

"My familiarity with BSC-POST has given me confidence that I can successfully use commercially available programs to program $\mathrm{CNC}$ milling machines in a 'real-world' industrial setting."

The cost to Buffalo State College to date is $\$ 0$.

\section{Conclusions and Future Research}

The "beta" version of BSC-POST is ready for incorporation into the CAD/CAM course this spring. The program has met all of the objectives that it could meet up to this date. Initial results based on the outcomes of the exams and projects, as well as the responses to the questionnaire, will be available for presentation in Nashville in June.

The Department of Technology will be moving to a new facility in the near future. It is anticipated that funding for laboratory equipment will be available for the new space acquired, and thus additional CNC machines will be purchased. Modules for these machines will then have to be incorporated.

Some of the machines currently here have 3D capability. VAL-CAM will eventually obtain this capability, at which time, in parallel, BSC-POST must obtain this capability as well. Not only will the presence of a third dimension increase the complexity of those modules that should have 3D capability, it will increase the graphical manipulation requirements of BSC-POST as well.

The importance of student involvement will not be overlooked. Suggestions made by students will be taken very seriously and incorporated as required. Those students talented enough to participate in the code development process will be invited to do so.

\section{Acknowledgement}

The authors would like to thank Scott Greenberg, a mechanical engineering technology student at Buffalo State College, class of 2003, for his assistance in this project. 


\section{Appendix: Program Overview}

BSC-POST is designed with the following points in mind:

- The program should be modular, where each module consists of machine dependent properties and methods. This would facilitate expansion of the program as one added new machines.

- The program should have a graphical user interface so that the user can visually determine the geometry utilized in the process and evaluate choices concerning the process.

- The program should be user friendly, and fully understood by engineering technology students familiar with beginning computer aided manufacturing.

To these ends, it was decided that the program should be written in Visual Basic. It would have the Windows "look and feel". It would display the geometry of the process based on the input file generated by VAL-CAM. Interaction with this geometric display would depend on what was discovered in the process of researching each machine. Development of image manipulation (zooming, rotating, panning, etc.) would be postponed in favor of development of functionality, particularly since the capabilities of VAL-CAM at this time are limited to two-dimensional machining.

The program flow is as follows:

- A form containing a picture box and a drop down menu appears (Figure 1). A VALCAM output file is selected and read in. See Figures 2 and 3.

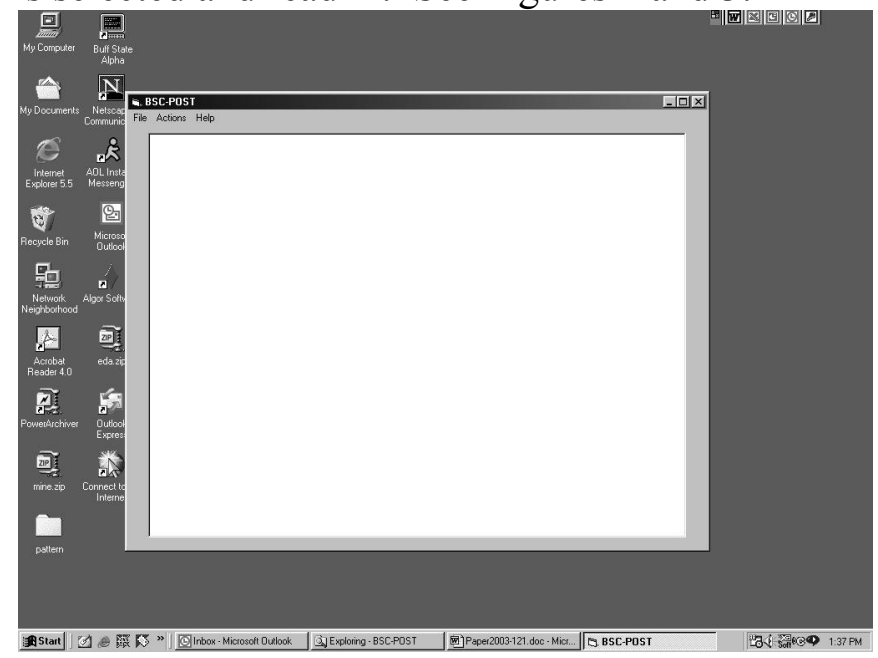

Figure 1: BSC-POST Opening Screen 


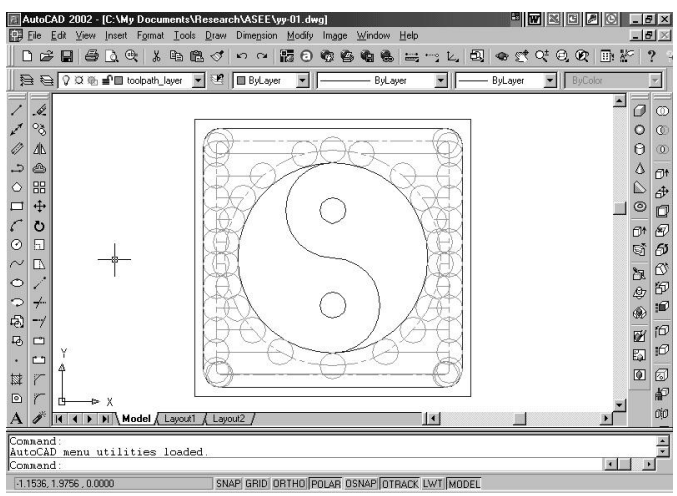

Figure 2: VAL-CAM Output Displayed in AutoCAD

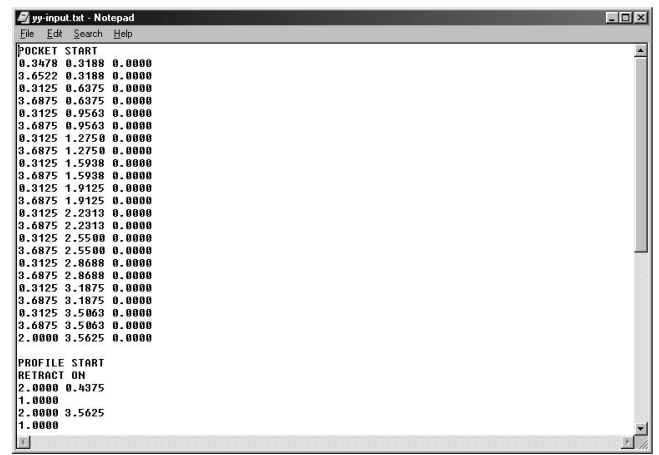

Figure 3: Text File Displaying VAL-CAM Output/BSC-POST Input

- The user is then chooses a machine from a drop-down list (Figure 4). Once chosen, the user can then select machine specific properties from a machine specific form (Figure 5).

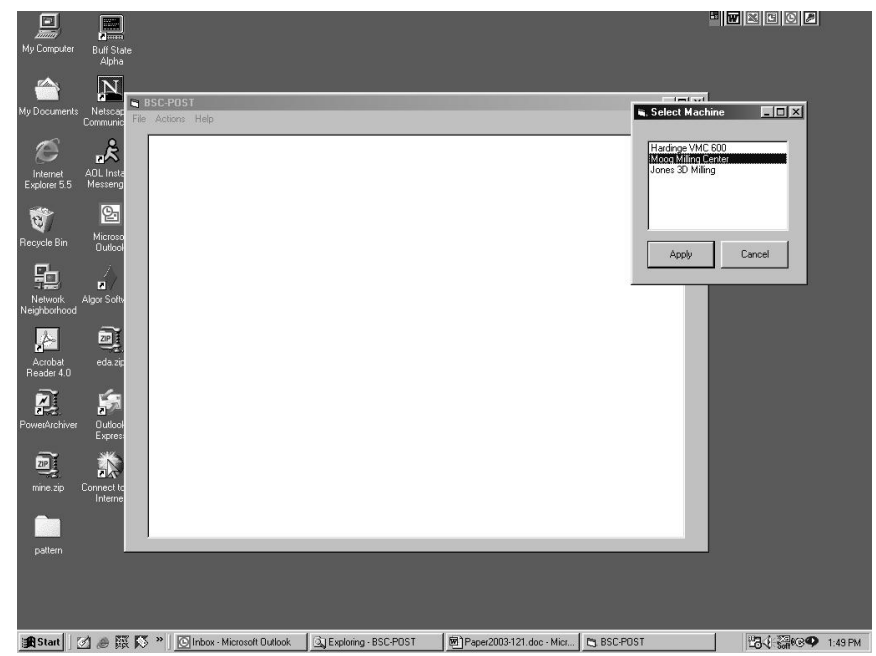

Figure 4: Choosing the Machine 


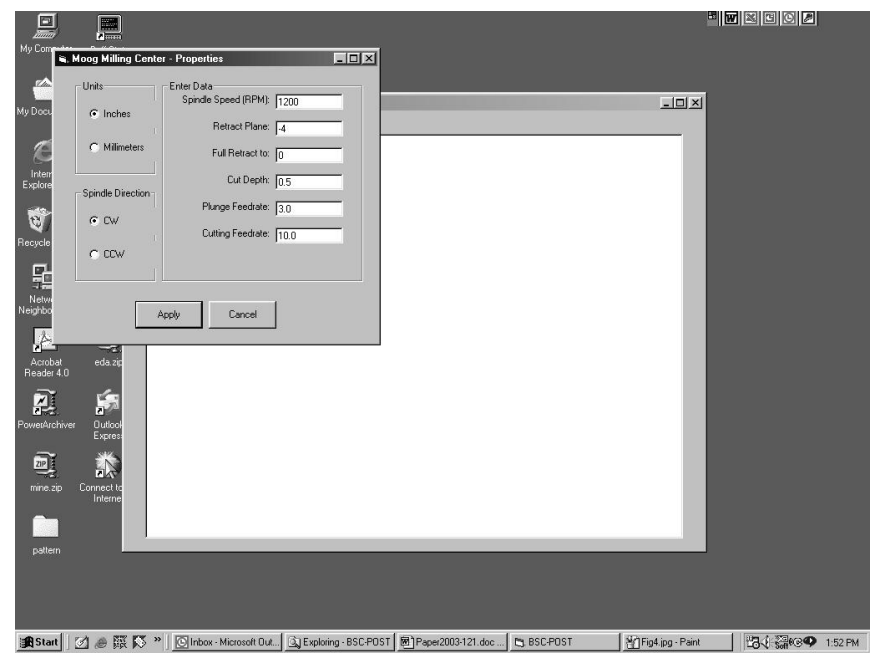

Figure 5: Assigning Additional Machine Properties

- After the user is satisfied that all options have been assigned, the user gives a command to generate the CNC code to be stored in a file (Figure 6). The file can then be displayed and checked. In addition, a graphical representation of the machining process appears on the main form (Figure 7). If correct, the process is complete; if not, further assignment of options would be possible.

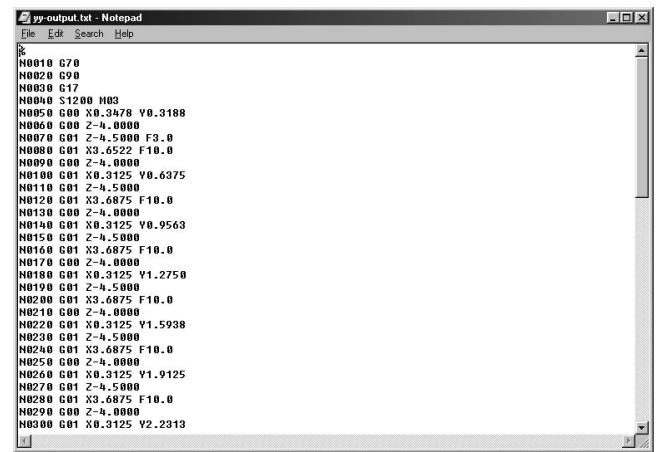

Figure 6: BSC-POST Output File 


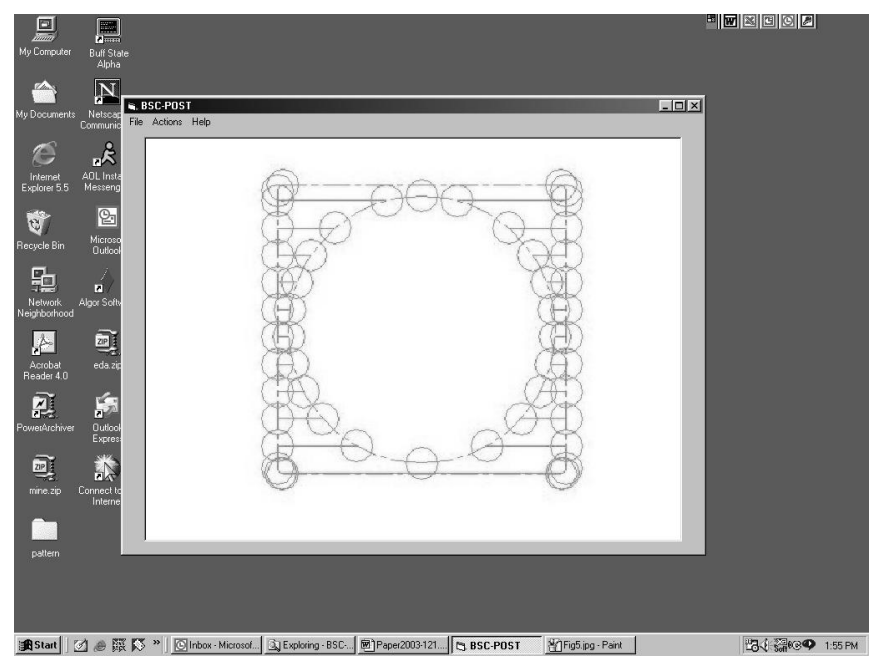

Figure 7: Graphical Output of Machining Process

\section{Bibliography}

1. A. Hotchkiss, "VAL-CAM, the Use of Computing Technology in Computer-Aided Manufacturing (CAM) Curriculum", Proceedings of the $5^{\text {th }}$ SUNY Conference on Instructional Technologies, Oswego, May 21-24, 1996.

2. A. Hotchkiss, "Development of a Software Program, VAL-CAM, Used in a Computer-Aided Manufacturing (CAM) Curriculum for Contour Milling Processes", Proceedings of the $5^{\text {th }}$ SUNY Conference on Instructional Technologies, Oswego, May 21-24, 1996.

3. A. Hotchkiss, "The Development of a Profile Milling Program for Teaching Computer-Aided Manufacturing and CNC Programming", ASME Proceedings of the $17^{\mathrm{th}}$ Annual International Computers in Engineering Conference, September 14-17, 1997.

\section{Biographical Data}

SLADE GELLIN received his Ph.D. from Harvard University in 1977. Since that time he has spent his career in both industry and academia developing engineering software. He is currently an assistant professor of mechanical engineering technology.

ANTHONY HOTCHKISS received his Ph.D. from Southampton University, England in 1977. He has worked in the aerospace and computer industries before entering academia in 1987. He has more than 100 publications in $\mathrm{CAD} / \mathrm{CAM} / \mathrm{CAE}$, and is currently a professor of industrial technology at Buffalo State College. 